in experiments as well as in field studies, subjects' social integration (and especially social status gains as a consequence of acts of violence on the one hand and social skills and general intelligence on the other) should be controlled so that we can distinguish between the adaptive and nonadaptive, that is, the smart and the dumb kinds of aggressiveness. Perhaps then we will observe a positive correlation between measured $\mathrm{T}$ and smart aggressive behavior, just as there is a positive correlation between $\mathrm{T}$ and adaptive (successful) competitive behavior.

\section{Placebo-controlled manipulations of testosterone levels and dominance}

Ronal E. O'Carroll

Psychology Department, University of Stirling, Stirling, FK9 4LA Scotland, United Kingdom. reo1@stir.ac.uk

\begin{abstract}
M azur \& Booth present an intriguing model of the relationship between circulating testosterone levels and dominance behaviour in man, but their review of studies on testosterone-behaviour relationships in man is selective. Much of the evidence they cite is correlational in nature. Placebo-controlled manipulations of testosterone levels are required to test their hypothesis that dominance levels are testosteronedependent in man. The changes in testosterone level that follow behavioural experience may be a consequence of stress. Testosterone levels in man are determined by a wide variety of factors, and a multivariate approach is required.
\end{abstract}

Mazur \& Booth $(M \& B)$ propose a reciprocity between testosterone $(T)$ and dominance behaviour in man. The emphasis on "bidirectionality" (H atch 1981) is to be applauded. H owever, their review of the literature is highly selective. They refer extensively to positive findings that do not appear to have been subject to the normal peer-review process necessary for publication in scientific journals (e.g., B ooth \& D abbs 1995; D abbs \& H argrove, in press; Fielden et al. 1994; M azur \& M ichalek 1995; M azur et al., in press), and they neglect to cite published negative findings. F or example, O'C arroll and B ancroft (1984) reported a placebocontrolled study in which circulating $T$ levels were manipulated in endocrinologically normal men, and no change in aggressive mood state was observed. In a further study of men with low circulating $\mathrm{T}$ levels, elevation of their $\mathrm{T}$ levels, in a placebo-controlled manner, resulted in a reduction in self-rated aggressive and irritable mood, if anything ( $O^{\prime} C$ arroll et al. 1985). F inally, in a placebo-controlled single-case study, O 'C arroll \& B ancroft (1985) described the case of a mentally handicapped young man who had been castrated traumatically in a road accident in childhood. He required $\mathrm{T}$ injections in order to stimulate epiphyseal closure (cessation of long bone growth). However, the nursing staff demanded that the injections should be stopped as they were apparently causing hyperaggressive behaviour. When varying doses of oral T were evaluated using a placebo-controlled, doubleblind design, nurse ratings failed to detect any significant effect on aggressive behaviours. Such experimental designs, although difficult, are required in order to determine hormone-behaviour relationships in man.

Aggressive behaviour is clearly not "one thing"; it is likely that only some aspects of aggression may be related to $T$ levels. Response to provocation or threat appears to be a promising candidate, worthy of further investigation, particularly insofar as positive results have been obtained from a variety of sources: adolescence (Olweus et al. 1980; 1988), the laboratory (K ouri et al. 1995), and sporting behaviour (Scaramella \& Brown 1978).

$M \& B$ propose in section 1 that it would be "naively behavioristic" to deny our ability to read people's intentions. However, particularly in relation to aggressive behaviour, we must acknowledge that people often misperceive the social signals and inten- tions of others ( $\mathrm{N}$ avaco 1986). Aggressive men often misperceive. F or example, an innocent glance may be construed as a challenging gaze (inferred malevolence where none exists), thus leading to challenges and overt aggression.

In section 1 it is proposed that dominance mechanisms have clear evolutionary advantage. I t is interesting to note recent claims for the opposite viewpoint, for example, that submissiveness as a personality trait is associated with protection from coronary artery heart disease or that submissiveness not dominance confers survival advantage (Whiteman et al. 1997).

$M \& B$ correctly point out that $T$ levels in man fluctuate markedly (sect. 2). This fact makes interpretation of correlational studies extremely difficult, particularly in that most studies have relied on single-sampling methodology. $M \& B$ cite reliability values in the region of $r=.5$ as proof of within-subject consistency of $T$ levels across years (sect. 9). This is not particularly impressive as a reliability figure; $T$ level at time 1 predicts $25 \%$ of the variance in T level at time 2, leaving $75 \%$ of the variance unexplained. Given this degree of within-subject variation, claiming hormonebehaviour relationships based on single-sample correlational methodology may not be warranted.

In their discussion of reciprocal causation (sect. 5), $M \& B$ propose that a precompetition boost in $T$ level would make individuals more "sensation seeking" and willing to take risks. In support of this view, they cite Daitzman and Zuckerman (1980), who described a correlation between sensation-seeking behaviour and gonadal hormone level. M \&B fail to cite O 'Carroll (1984), however, who showed that placebo-controlled manipulations of circulating $T$ levels in groups of both eugonadal and hypogonadal men had no effect whatsoever on any measure of sensationseeking behaviour.

$M$ uch is made of reductions in circulating $T$ levels following adverse experience (sect. 5), and the hypothesis is proposed that these changes reflect changes in dominance levels. However, surely the most parsimonious explanation (as $M \& B$ admit in their caveat in sect. 8) is that these changes are due to stress effects, not status loss. The seminal study in this field, cited in support of the dominance theory, is in fact entitled "Suppression of plasma testosterone levels and psychological stress" (K reuz et al. 1972).

$M$ uch is made of dominance contests as well. I for one remain unconvinced that normal day-to-day social interaction is filled with such confrontations (sect. 7). The examples given (e.g., length of stare leading to stress in the recipient) would surely be expected to lead to alterations in the hypothalamic-pituitary-adrenal axis rather than the gonadal steroid level.

It is rather worrying that a number of sweeping generalisations are made without supportive evidence, for example, the claim of elevated $T$ levels in black males "possibly reflecting the higher defensive demands on black men during young adulthood" (sect. 8) and the claim that "normal marriages are secure and supportive, more free from stress than single life" (sect. 9).

The evidence regarding divorce, marriage, and T (sect. 9) is particularly difficult to evaluate. The U.S. Air F orce data to which $M \& B$ refer is supported by two references, one unpublished and one in JAM A. M \&B describe 2,100 U.S. Air F orce veterans, yet the cited JAM A paper describes 995 veterans who were exposed to herbicides during aerial spraying of "Agent Orange" in Vietnam versus 1,299 comparison subjects. $M \& B$ report significant correlations between $T$ level and marital status, claiming that 10 of 16 correlations were "significantly positive" and that $\mathrm{T}$ levels are highly responsive to marital status. H owever, T levels were apparently taken every 3 years, hence a man may have been divorced nearly 3 years prior to his T measurement and a multitude of life events and biological and psychological stressors may have occurred in the intervening period, all of which could conceivably have a significant effect on his endocrinological status. $M \& B$ claim that "T measured right after the divorce is the best predictor." What does "right after divorce" mean - some time within the preceding 3 years? If there is an association between high $T$ and recent divorce, could this not perhaps reflect elevation of circulat- 
ing $T$ level following cessation of prolonged marital stress? As $M \& B$ state, the break-up of a marriage usually spans years, "accompanied by arguments and confrontations" (sect. 9). Hormone levels are multiply determined, and choosing a single crude demographic measure such as marital status ("because there was little behavioral measurement in the study") and looking for endocrine associations is unlikely to lead to significant advances in our knowledge of endocrine- behaviour relationships in man.

In conclusion, the link between $\mathrm{T}$ and aggressive and sexual behaviour in lower animals (e.g., rodents) is clearly established. However, as we ascend the phylogenetic ladder to humans, this relationship becomes less clear. This is not to deny that such a relationship exists, but the complexity of human social behaviour suggests that both behaviour and endocrine status are influenced by a wide variety of biological and psychological variables, and a multivariate approach is required. $F$ urthermore, as a consequence of the pulsatile variability in circulating $T$ levels in man, significant error variance is introduced into single-sample correlational studies. Definitive evidence is likely to come from placebocontrolled, double-blind experiments in which circulating $\mathrm{T}$ levels are manipulated and appropriately reliable and sensitive assays of behaviour are taken.

\section{Of fish and men: A comparative approach to androgens and social dominance}

\author{
Rui F. Oliveira \\ Unidade de Investigação em Eco-Etologia, Instituto Superior de Psicologia \\ Aplicada, 1100 Lisboa, Portugal. ruiol@ispa.pt
}

\begin{abstract}
F our aspects of M azur \& B ooth's target article are discussed from a comparative perspective using teleost fish as a reference: (a) the relationship between aggression, dominance, and androgens; (b) the interpretation of the data in light of the challenge hypothesis; (c) the potential role of testosterone as a physiological mediator between social status and the expression of male characters; and (d) the fact that metabolic conversions of testosterone may be important in its effect on aggression/dominance.
\end{abstract}

As a fish biologist interested in hormones and behavior, I was fascinated to find myself to some extent on very familiar ground while reading $M$ azur $\&$ Booth's ( $M \& B$ 's) target article on dominance and testosterone in humans. However, insofar as testosterone ( $\mathrm{T}$ ) in particular and androgens in general are an essential part of the conserved vertebrate reproductive axis (i.e., hypothalamus-pituitary-gonads), one would expect some parallels between teleosts and mammals, including humans, in the relationships between sex hormones and social behavior (although $M \& B$ neglect to acknowledge the comparative literature on androgens and dominance in nonprimate vertebrates). $\mathrm{H}$ ere I will comment on four issues raised by $M \& B$ in the light of this comparative approach, using the teleosts as counterpoint. Teleost fishes are the most diverse of living vertebrate taxa and represent a very successful lineage of recently evolving organisms (N elson 1994). It would accordingly be very interesting to compare hormone-behavior systems in these two successful vertebrate lineages.

Dominance, aggression, and androgens. In the target article, $M \& B$ point out that dominance in humans may be exerted nonaggressively and that $T$ is related primarily to dominance and not to aggression per se, except when dominance is asserted aggressively. They go on to suggest that nearly all primate studies linking $T$ to aggressive behavior can also be seen as linking $T$ to social dominance.

In fish, castration lowers both androgen and aggression levels but not social dominance (F rancis et al. 1992). These results can be explained by the fact that aggression is an individual attribute, whereas social dominance is a relational one, which can vary with the social context into which the individual is placed (Bernstein
1981; F rancis 1988). [See also Bernstein: “D ominance R elationships and Ranks" BBS 3 1981.] M oreover, the underlying mechanisms involved in dominance relationships may differ according to the number of individuals involved. I n dyads, dominance may be more directly related to aggression, because the two individuals are competing directly, whereas, in triads, other phenomena may be involved, such as prior experience, individual recognition, bystander effects, or transitive inference. It would accordingly be expected that, in cases in which dominance is assessed in a dyad, $T$ can be more easily related to aggression. $N$ evertheless, $T$ is also known to be related to attention/cognitive mechanisms ( $\mathrm{H}$ ampson \& Kimura 1992), which might also be involved in statusassessment processes; thus T could still be linked to dominance in this scenario. The findings linking $T$ to aggression but not to social dominance in fish can thus be explained as a resilience effect of the dominance relationship previously established between each pair of tested individuals. I t would therefore be instructive to pay more attention to the context in which the data are collected and to the possible underlying mechanisms involved in status acquisition, in considering the relationship between $T$ and dominance.

The challenge hypothesis. Wingfield (1984) has proposed that the androgen levels of a given individual will respond in the short term to the social interactions in which the animal has participated, which will result in an adjustment of the readiness and intensity of the agonistic behavior according to changes in the social environment into which the animal is placed. In this view, variation in T levels may be more closely associated with temporal variations in aggression than with basal reproductive physiology. According to the challenge hypothesis, baseline breeding levels of $T$ are sufficient for normal reproductive function and temporal patterns in T levels may differ between species according to the mating system of the population. In monogamous species, T levels should rise above the baseline breeding level only in periods of social challenge, so that aggression will not interfere with parental care and pair bonding, whereas, in polygynous species, $\mathrm{T}$ levels should increase to near the maximal level and remain high, because this will facilitate aggressive behaviors in male-male competition (Wingfield et al. 1990). As the human species is considered to be monogamous and does not present a breeding seasonality, the challenge hypothesis would predict human male T levels to respond sharply to social challenges. In fact, the data presented by $M \& B$ provide further evidence for the challenge hypothesis; $T$ rises in response to a competitive match, as if in anticipation of the challenge. This precontest rise in $T$ might have the function of preparing the individuals for confrontation by increasing readiness to fight and improving the cognitive capabilities required by a competitive situation.

Again, there are parallel data for teleosts. Socially isolated males show low levels of both aggression and plasma androgens; these levels increase very rapidly after visual exposure to a territorial male, which acts as a challenge stimulus ( $H$ annes \& F ranck 1983; Heiligenberg \& Kramer 1972). Territorial males have higher androgen levels than nonterritorial males, and recently established territorial males undergo a large increase in androgen levels. After territory establishment, androgen levels drop to the territorial male baseline. F urthermore, simulated territorial intrusions promote an increase in androgen levels in resident males (Barnett \& Pankhurst 1994; Cardwell \& Liley 1991; Oliveira et al. 1996). This link between androgens and social status has also been shown to be a function of the number of territorial intrusions and of population density (Pankhurst $\&$ Barnett 1993). These data suggest that short-term increases in circulating androgens are a response to intense social competition during territory establishment. I t must be of high adaptive value to react to the presence of a male intruder with a quick rise in agonistic motivation, which might be achieved by high androgen levels. Subordinate individuals should adjust their aggressive behavior to a level that maximizes their reproductive success without promoting excessive confrontations with dominant males. This trade-off may be regulated by social modulation of androgen levels. 